\title{
Impacto de la organización del trabajo en los procesos formativos del sector automotriz argentino*
}

\author{
Delfini, Marcelo** \\ Pujol, Andrea*** \\ Roitter, Sonia****
}

\begin{abstract}
Resumen
El presente trabajo tiene como propósito estudiar la vinculación existente entre las formas de organización de la producción y el desenvolvimiento de los saberes laborales de los trabajadores de empresas terminales y proveedoras automotrices, considerando en qué medida ciertos dispositivos contribuyen a incrementar el saber del trabajador. La metodología utilizada fue fundamentalmente cuantitativa, a partir de los datos relevados en una encuesta realizada a los trabajadores de la trama automotriz durante el año 2006 que se complementó con técnicas características de las estrategias cualitativas. A partir de indicadores vinculados a las formas organizacionales se realizó la construcción de grupos homogéneos (cluster) los cuales muestran las condiciones bajo las cuales los operarios realizan sus tareas. Entre las conclusiones se destaca la existencia de una heterogeneidad de situaciones en torno a la organización del trabajo, lo que permite superar la dicotomía entre el modelo taylorista-fordista y los modelos de "lean production", observándose procesos más virtuosos entre los trabajadores de las empresas terminales.
\end{abstract}

Palabras clave: Organización del trabajo, aprendizaje laboral, economía del conocimiento, trama automotriz.

Recibido: 08-07-08. Aceptado: 10-12-09

* El presente trabajo se realizó en el marco del proyecto "Tramas Productivas, Innovación y Empleo en la Argentina" (PAV 057) Dirigido por Gabriel Yoguel, financiado por la Agencia Nacional de Promoción Científica y Tecnológica (Secyt), desarrollado en la Universidad Nacional de General Sarmiento (UNGS) y en otras universidades y centros del país.

** Investigador docente de la Universidad Nacional de General Sarmiento y Docente de la UBA, E-mail: mdelfini@ungs.edu.ar.

*** Docente-Investigadora de la Cátedra de Psicología Laboral. Facultad de Psicología. Universidad Nacional de Córdoba. E-mail: apujol@psyche.unc.edu.ar

**** Investigadora docente de la Universidad Nacional de General Sarmiento.

E-mail: sroitter@ungs.edu.ar 


\title{
The Impact of Labour Organization on Educational Processes in the Argentine Automotive Sector
}

\begin{abstract}
The purpose of this paper is to analyze existing connections between production organization forms and the development of workers' knowledge in carmakers and their suppliers, considering the extent to which certain devices contribute to increasing worker knowledge. The methodology used was fundamentally quantitative, based on data collected during interviews with 116 workers in 2006, complemented with techniques characteristic of qualitative strategies. Based on indicators connected to the organizational forms, homogeneous groups (clusters) were formed which showed the conditions under which the operators perform their tasks. Conclusions highlighted the existence of heterogeneous situations in terms of labour organization, which makes it possible to overcome the dichotomy between Taylor-Ford and lean production models, observing more virtuoso processes among workers at the carmakers.
\end{abstract}

Key words: Labour organization, worker learning, knowledge economy, automotive area.

\section{Introducción}

Las transformaciones desarrolladas en los últimos treinta años en la economía mundial sugieren la centralidad del conocimiento y la innovación en la competitividad dinámica, no sólo a nivel de las empresas, sino también dentro de las redes de las que éstas forman parte (Erbes et al., 2006; Arundel, 2003; Lundvall, 2009 entre otros).

La concreción de estos cambios exige que las empresas estimulen los procesos de aprendizaje con el fin de incrementar los saberes de los trabajadores y de producir mejoras en la productividad. De esta forma, las firmas se constituyen en organizaciones formativas y se establece una relación dinámica entre formación y productividad. En este sentido, las formas de organizar el trabajo que tienden a estimular procesos de aprendi- zaje a partir de su estructura organizativa se asemejarían a las que caracterizan al modelo de producción japonés: trabajo en equipo, polivalencia y participación activa de los trabajadores, entre otros elementos (Arundel et al., 2007; Lorenz y Valiere, 2005, Zarifian, 1995).

En este marco, el presente trabajo tiene como propósito indagar sobre la vinculación existente entre las formas de organización de la producción y el desenvolvimiento de los saberes laborales de los trabajadores, considerando en qué medida ciertos dispositivos organizacionales contribuyen a incrementarlos. En este sentido se ofrece una caracterización de las formas de organización del trabajo prevalecientes en la trama automotriz, considerando las principales terminales y sus proveedoras.

El artículo presenta algunas respuestas a los siguientes interrogantes: 
Impacto de la organización del trabajo en los procesos formativos del sector automotriz Delfini, Marcelo; Pujol, Andrea y Roitter, Sonia

¿qué formas de organización del trabajo favorecen los procesos de aprendizaje y la generación de capacidades laborales en los trabajadores?, ¿qué tan presentes se encuentran estas formas de organización del trabajo en las empresas de la trama estudiada?, ¿qué perfil de operario puede desarrollarse en el marco de las formas de organización del trabajo prevalecientes actualmente en las empresas?, ¿existen diferencias entre las empresas que integran la trama estudiada según la posición que ocupan en la misma?

La elección de la trama automotriz para la realización del presente análisis y la mirada puesta sobre los operarios de las firmas, obedece a la importancia que tiene esta trama en la actividad industrial, tanto a nivel mundial como en Argentina, lo que lleva a convertirlo en un sector muchas veces paradigmático y ejemplificador de cambios, incorporación de nuevos dispositivos tecnológicos, empleo y condiciones de trabajo. En efecto, las terminales y sus proveedoras explicaban para el 2005 el $5,9 \%$ del VAB manufacturero y el $4,8 \%$ del empleo en la industria. La importancia de la trama se observa también, en el crecimiento experimentado por la producción entre el 2003 y el 2007 que alcanzó un $34 \%$ promedio anual, en tanto el incremento de la productividad pasó de 15 vehículos por persona ocupada en 1994 a 22 en 2006.

Por otra parte, en la industria automotriz se han realizado numerosos estudios sobre las formas de organización del trabajo y además se ubica a las terminales como elemento ejemplificador, tanto de los cambios tecnológicos como de la gestión social de las empresas, tanto en Argentina como en el orden internacional.
Según estos estudios, durante la década de 1990 se llevaron adelante en la industria automotriz radicada en el país $\mathrm{y}$, en mayor medida, en las terminales procesos de cambio con la incorporación de las lógicas de producción ligera o flexible, los cuales no se han desarrollado de la misma manera en el conjunto de la trama (Motta, et al. 2007, Roitter, et al., 2008, Yoguel y Novick, 2000). Sin embargo, estos estudios no han podido dar cuenta de la profundidad de dicha modernización y de su alcance y efectividad en lo relativo a la implicación de los trabajadores y a los beneficios percibidos.

En el caso de este análisis, se puso en foco la situación de los operarios de la trama, considerando para ello las empresas terminales y proveedoras, clasificadas en globales y no globales. Las primeras, se caracterizan por abastecer de conjuntos a las terminales y por un nivel de cercanía mayor a éstas que el resto de las empresas, puesto que su alcance corporativo les permite seguir a las terminales donde éstas se instalen. A fin de identificarlas, en este estudio se consideró como globales a las empresas pertenecientes a las 100 autopartistas con mayor facturación a nivel mundial. Las no globales, por su parte, constituyen el resto de los proveedores nacionales y multinacionales.

La metodología utilizada fue fundamentalmente cuantitativa y se basó en datos obtenidos en una encuesta aplicada a trabajadores operativos durante el periodo mayo- diciembre de 2006. La misma fue realizada a 116 trabajadores de los cuales 28 pertenecen a firmas terminales, empresas que conforman los núcleos de la trama, 39 pertenecientes a 
9 firmas proveedores globales y 49 operarios de 17 proveedores no globales ${ }^{1}$. Junto con los resultados arrojados por la encuesta, se dará cuenta del análisis de las entrevistas en profundidad, realizadas a trabajadores de las terminales y de proveedoras. Con ello se intenta ahondar en algunos aspectos del estudio.

La información provista por la encuesta permitió elaborar indicadores vinculados al trabajo en equipo, polivalencia, participación y autonomía en la realización de tareas. Luego se efectuó un análisis factorial de correspondencias múltiples (AFCM) que permitió constituir grupos homogéneos (Cluster) considerando simultáneamente los diferentes planos que determinan la forma de organización del trabajo prevaleciente. La aplicación de esta técnica posibilitó, además, la construcción de grupos homogéneos (clusters) que evidencian las condiciones organizacionales bajo las cuales trabajan los operarios de la trama automotriz.

En lo que sigue se plantean, en primer lugar, las principales proposiciones teóricas vinculadas a la temática. En la segunda parte, se explicita la metodología utilizada, los indicadores considerados para el procesamiento de la información relevada y los resultados obtenidos en el análisis de datos. Finalmente, se presentan las conclusiones más relevantes del estudio y se plantean algunos interrogantes con el fin de abrir otras exploraciones.

\section{Innovación, conocimiento y aprendizaje}

La problemática puede situarse en el contexto de un capitalismo de innovación intensiva (Hatchuel y Weil, 1999) que pone en crisis los modos tradicionales de producción y renovación del saber. En este marco, se tornan cuestionables los principios clásicos que asignaban un papel secundario al aprendizaje en la dinámica económica y se produce, en contrapartida, una puesta en valor del conocimiento como factor de producción, soporte de los procesos de innovación y a su vez producto inherente a la economía (Lundvall, 2003). Así, se resalta la idea de que las empresas y las comunidades de prácticas -y sus interacciones- juegan un papel central en los procesos de producción de conocimiento (Erbes et al, 2006; Nelson y Winter, 1982; Rullani, 2006) $)^{2}$.

Las articulaciones entre conocimiento y procesos de innovación se producen a nivel organizacional a través de

1 La muestra es no probabilística y para la realización de las encuestas se consideraron dos criterios para minimizar sesgos en las respuestas: el primero fue que la realización de las encuestas no estuviese determinada por ningún actor de los que intervienen en las relaciones laborales, esto es, ni por la empresa ni por el sindicato. El segundo criterio, dada la categorización realizada, consistió en expandir la muestra a un número de empresas relevantes y no concentrarse en unas pocas firmas.

2 Nos referimos aquí a la innovación en transformación de una idea en un producto nuevo o mejorado que es introducido en el mercado, o en un proceso de fabricación nuevo o significativamente mejorado que es utilizado en la industria. 
Impacto de la organización del trabajo en los procesos formativos del sector automotriz Delfini, Marcelo; Pujol, Andrea y Roitter, Sonia

la gestión de los procesos de creación de conocimiento en la empresa. En este plano, los conceptos de rutinas e innovación constituyen componentes centrales a la hora de teorizar sobre los procesos de aprendizaje. Las organizaciones regulan el comportamiento de los diversos agentes a través de sus normas. La interacción entre la adecuación a las rutinas, el quiebre de las mismas y el establecimiento de nuevas rutinas es un aspecto fundamental del aprendizaje (Nelson y Winter, 1982; Arocena, 1996).

En este sentido, las empresas no sólo deben crear procedimientos y mecanismos orientados a compartir experiencias, sino que también deben generarse situaciones de reflexión sobre aquello que se hace, y esto incluye la necesidad de que los individuos puedan ir mas allá del mero intercambio, cuestionando las estrechuras profundas y performances de un cierto contexto organizacional (Rullani, 2006).

En los desarrollos clásicos del aprendizaje organizacional (Argyris y Schon, 1978) se destaca la distinción entre el aprendizaje de primer orden y el aprendizaje de segundo orden. Según estos autores, el aprendizaje de primer orden o primer ciclo trata de mejorar la capacidad de la organización para alcanzar objetivos conocidos y está estrechamente vinculado al aprendizaje de rutinas. EI aprendizaje de segundo orden, está signado por la reflexividad que vuelve a evaluar la índole de los objetivos, así como los valores y las creencias que los sostienen y fundamentan; lo que ofrece un camino fértil para los procesos de innovación: una organización que aprende es aquella capaz de crear, adquirir y transfe- rir conocimiento, modificando su comportamiento en función de ese aprendizaje.

En relación a los mecanismos que hacen posible la creación de conocimiento organizacional, Nonaka y Takeuchi (1999) señalan que éstos se apoyan en la interacción entre conocimiento tácito (experiencial, no codificado) y explícito (codificado o formal), y que se encuentran estrechamente ligados al "diseño" de la organización, y particularmente de la organización del trabajo, y a la aplicación de un conjunto de políticas de gestión que operen como condiciones facilitadoras de aprendizaje. En este sentido se trata de llevar adelante procesos de aprendizaje compartido, que no queden limitados a una minoría pensante (Montero Leite, 1996).

De esta manera, puede reconocerse la existencia de dos grandes corrientes en torno al desarrollo de los procesos de aprendizaje en los espacios de trabajo. La primera de ellas se vincula a la idea de "empresa que aprende" y a las formas organizacionales fundamentadas en la ciencia-acción. En esta perspectiva, la empresa en su conjunto se constituye en un sistema capaz de aprender a través de la articulación de los procesos de aprendizaje que se desarrollan en las instancias de trabajo. Se asumen dos enunciados: los procesos de aprendizaje se vincularían a las estructuras organizacionales que permiten una circulación recurrente de la información y una constante explicitación del conocimiento; además, el aprendizaje "de la organización" resulta más significativo que el aprendizaje de cada uno de los actores que intervienen en ese espacio.

Otras corrientes, en cambio, señalan que las posibilidades formativas radi- 
can en las experiencias concretas que ofrece el espacio de trabajo, en las relaciones entre los trabajadores y el proceso de producción. En este sentido, no es la organización "toda" la que posibilita este desarrollo sino el carácter calificante del trabajo, que emerge del análisis de la organización del trabajo y no del funcionamiento interno de la empresa. La expresión más certera de esta conceptualización sostiene que "cada actor de la empresa es productor de conocimiento y de competencias, en tanto exista un dispositivo que favorezca esta creación y un proceso que lo capitalice (...) es el trabajo que es formador, que hace aprender y es calificante; no lo es la organización ni la empresa" (Parlier, 1996). La idea que subyace en estos enunciados privilegia los conocimientos tácitos devenidos de las prácticas productivas.

\section{Organización del trabajo, innovación y desarrollo de capacidades}

Las dos formas de concebir los procesos de producción del conocimiento que hemos enunciado, pueden articularse a través de la noción de "organización calificante" (Zarifian, 1996 y Montero Leite, 1996). Esta noción evidencia las formas en que se procesan los conocimientos en las instancias de trabajo y el modo en que interviene la organización del trabajo en la creación de nuevas capacidades y conocimientos, generando un proceso virtuoso entre organización del trabajo y competencias.

En esta perspectiva, el impacto de la organización del trabajo en la formación y desarrollo de capacidades de los trabajadores depende tanto del diseño de las actividades y su organización, como de la gestión de las rutinas y las innovaciones. En este sentido, juegan un papel importante algunos componentes clave de la gestión que dan "contenido" al modo de organizar el trabajo: los criterios de asignación de tareas, la implementación de dispositivos grupales que permitan la conversión de conocimiento y la reflexión sobre la acción, la producción colectiva de rutinas e innovaciones, entre otros.

La organización debe crear las condiciones para el despliegue del aprendizaje al menos en dos sentidos: por una parte, a través de un contexto apropiado para facilitar la participación de los individuos en diferentes actividades que impliquen interacción grupal; por otra parte, se deben diseñar dispositivos de interacción que se constituyan en espacios de participación, cooperación y transmisión de conocimientos de los individuos al resto de la organización.

Esta perspectiva se ve enriquecida a través de la noción de organización formativa que refiere a las posibilidades de aprendizaje que ofrece el ambiente organizacional a través de su estructura o diseño (Zarifian, 1996). Así, al interior de las firmas, los procesos formativos sirven para incrementar las competencias laborales si se sustentan, fundamentalmente, en las propias instancias de trabajo y parten del mismo proceso de producción. De esta manera, el aprendizaje en el puesto de trabajo es un proceso multidimensional que se puede definir con más detalle teniendo en cuenta las distintas dimensiones de las actividades y situaciones laborales. La organización del proceso laboral (cooperación y coordinación) de- 
Impacto de la organización del trabajo en los procesos formativos del sector automotriz Delfini, Marcelo; Pujol, Andrea y Roitter, Sonia

sempeña un importante papel de estímulo y apoyo a la adquisición de competencias (Gore, 2003).

De esta manera pueden establecerse estructuras específicas de organización del trabajo orientadas a favorecer la dinámica de la formación. En este sentido, una empresa es formativa y generadora de conocimiento en la medida que promueve procesos de aprendizaje en su interior a través de la participación de los trabajadores y de la interacción entre ellos (Mallet, 1995; Mertens, 2002; Méhaut, 1994; Zarifian 1995; Harteis, 2003).

Siguiendo estas tendencias, el discurso del management enfatiza las bondades otorgadas por las nuevas formas de organización del trabajo en el marco del modelo de producción ligera, como así también en las formas de autonomía vinculadas al "empowerment", cuestiones que son también señaladas como clave por la literatura managerial.

Desde otras perspectivas se pone en evidencia que estás lógicas de acción organizacional vinculadas al modelo japonés de producción parecen alejarse del mismo, en tanto existe una distancia entre el modelo y la realidad, entre los enunciados gerenciales y lo que se observa en el piso de la fábrica (Novick, 2000). También Linhart (2002) señala que la brecha que separa "discursos" de "prácticas" resulta notable. En el caso de América Latina, es preciso interrogarse acerca de las bondades sociales del modelo, ya que diversos estudios evidencian que su aplicación muestra, con frecuencia, efectos adversos focalizados esencialmente en el empeoramiento de las condiciones de trabajo y la desarticulación de las identidades profesionales
(Antunes, 2006; Mello e Silva, 2004). En síntesis, algunas experiencias de investigación y de práctica profesional indican que, en los procesos de reconversión productiva, las empresas muestran comportamientos heterogéneos (Yoguel, 2002), y las distintas realidades locales permiten la emergencia de "configuraciones hibridadas" (De la Garza Toledo, 1999; Novick, 2000) de los "modelos" enunciados en la teoría.

Desde la perspectiva del impacto en los trabajadores, Zarifian (1996) señala que los cambios que implican los nuevos modelos productivos sitúan a los actores en una posición de tensión. Se asiste a contradicciones de al menos dos órdenes: simbólicas y psicológicas. Los trabajadores se encuentran situados en el desempeño de roles en conflicto, en tanto el discurso que apunta a la subjetividad se traduce en palabras clave como "autonomía" y "responsabilización" en un universo organizacional aún altamente codificado en el que las posibilidades de toma de decisión son bastante limitadas y estandarizadas. Por una parte, la implementación de tecnologías (de producción, de información y de comunicación), de procedimientos muchas veces altamente prescriptivos y de un conjunto de herramientas de gestión estandarizadas (de la producción, de la calidad, de la productividad, de la seguridad e higiene, de los problemas e incidentes críticos, etc.) se orientan a hacer previsibles y controlables los escenarios de producción y tornan cada vez más estrechos los márgenes para el ejercicio de la autonomía. Por otra parte, prevalecen en la empresa sistemas de control de gestión que operan sobre las tareas pero muy rara vez sobre 
las coordinaciones entre actividades $y$, además, se apoyan significativamente en los mandos superiores inhibiendo el "empowerment", lo que es en sí contradictorio con la idea misma de autonomía y responsabilización (Zarifian, 1996).

Esta postura implica otorgar un papel central no sólo al carácter "calificante" de las organizaciones, a partir de la revalorización del aprendizaje y el conocimiento como factores de desarrollo, sino también al lenguaje y la comunicación como soporte de dichos procesos.

Se observa, entonces, que las interacciones comunicativas entre los trabajadores constituyen el componente central de los procesos de aprendizaje. En la perspectiva de Zarifian (1996) los cambios en el papel del lenguaje en la empresa -en su dimensión instrumental, cognitiva y social-, se articulan estrechamente con las transformaciones de los procesos de trabajo: trabajar juntos en las condiciones modernas de la producción es comunicar, en el sentido de construir y desarrollar un espacio de intersubjetividad y de comprensión recíproca.

Como se sabe, el lenguaje constituye un eje central en las reflexiones sobre el aprendizaje, en tanto los saberes tácitos o prácticos surgen de las experiencias e interacciones sociales cotidianas de las personas, que exigen el desarrollo de capacidades de entendimiento, comprensión, interpretación y aplicación.

\section{Las dimensiones de la organización del trabajo en la trama automotriz.}

Los enunciados teóricos de los apartados anteriores permiten afirmar que para pensar la influencia de la organización del trabajo en el desarrollo del aprendizaje se hace preciso identificar dos dimensiones: por una parte, la "arquitectura" o diseño organizativo del trabajo $\mathrm{y}$, por otra, las herramientas de gestión social del mismo.

En cuanto a las formas concretas en que el trabajo se organiza (la "arquitectura”), la tendencia al trabajo en células, redes y/o equipos de proyecto busca producir una mejora sustantiva en la comunicación e intercambio de saberes, y con ello en las coordinaciones de las actividades. No obstante, la mera estructuración grupal de las actividades no garantiza por sí misma el desarrollo de capacidades. Requiere, además, la puesta en juego de un conjunto de herramientas de gestión que "activen" dicha estructura en las prácticas: trabajo en equipo, gestión de polivalencia, promoción de autonomía e implementación de canales que hagan efectiva la participación de los trabajadores.

El trabajo en equipo conforma uno de los aspectos centrales de la dinámica formativa de las empresas. Por cuanto "lo grupal”, como herramienta social, permite que la actividad adquiera características enriquecedoras en la medida que promueve el intercambio de funciones entre miembros y la posibilidad de compartir experiencias a partir de la acción misma. No obstante, esta forma de organización requiere ciertas condiciones para poseer una lógica formativa: a) los equipos deben tener a cargo la realización de una parte del proceso de producción, en reemplazo del trabajo fordista realizado por medio de una línea de producción con asignación individual de tareas; b) los equipos deben tener una con- 
Impacto de la organización del trabajo en los procesos formativos del sector automotriz Delfini, Marcelo; Pujol, Andrea y Roitter, Sonia

formación multisectorial que, como en el caso de los equipos de mejora continua, permita un incremento de las capacidades mediante el intercambio entre pares.

En este estudio el indicador de equipo de trabajo refiere a la forma de realización de las tareas en el ámbito de la producción. En este sentido la realización de las tareas en equipos conformados por un número adecuado de miembros resulta virtuosa respecto de los procesos formativos. De esta manera, pudieron constituirse tres categorías para la realización de las tareas: Equipo virtuoso, Limitado o Individual, dependiendo de la forma de realización del trabajo (individual o colectiva) y la cantidad de integrantes del equipo. En este sentido, se han considerado equipos virtuosos a aquellos cuyos integrantes no superan el número de diez, mientras que la segunda de las categorías se encuentra conformada por aquellos equipos donde intervienen entre 10 y 20 trabajadores generando un espacio donde se limitan los procesos de intercambio. Por último, la categoría de carácter individual se encuentra conformada por aquellos casos donde las tareas se realizan por fuera de una célula o bien hay más de 20 integrantes en el equipo, lo cual supone una dinámica de trabajo que no permite los procesos de integración de experiencias $^{3}$.

El otro de los indicadores refiere a las características que asume la rotación en el espacio productivo, en el que se ponen de manifiesto diversas categorías relacionadas a la forma que adquiere la polivalencia. Al respecto, esta puede ser: enriquecedora, cuando se rota entre puestos de niveles de complejidad similar o superior y es programada por la empresa; de carácter funcional, cuando la rotación es programada pero se realiza entre puestos con distinto o menor nivel de complejidad o para cubrir cuellos de botella originados en la falta de personal, sin una planificación; por último, puede ser especializadora en la medida en que no se rota entre puestos. Este indicador permite establecer los rasgos estructurales y los dinámicos, ya que se analiza tanto la rotación como el carácter que esta adquiere.

Desde el punto de vista conceptual, la rotación se vincula directamente con la gestión de la polivalencia. Su análisis resulta útil para valorar la implicación de la conducción (en primer término de la supervisión) en el desarrollo de las capacidades de los trabajadores y de los equipos. La polivalencia es la capacidad que acredita un trabajador para desempeñarse hábilmente en un mínimo de dos puestos o dos funciones y un máximo de tres. La gestión de la polivalencia supone al menos tres actividades: a) relevar las capacidades o competencias requeridas por cada función productiva y los niveles de logro esperados; b) sistematizar dichas funciones en una matriz que permita "mapear" el capital humano de un área o sector y gestionar su desarrollo; c) evaluar las competencias de

3 La construcción de los indicadores que se mencionan en esta sección se basó en la información proveniente de entrevistas previas realizadas a informantes clave. 
manera periódica y decidir acciones que favorezcan en los trabajadores su adquisición o desarrollo.

Otro indicador contemplado en el estudio refiere a la autonomía. La autonomía suele ser considerada una competencia transversal, necesaria en la actualidad para la profesionalización de cualquier ocupación (Zarifian, 1996). Remite particularmente al posicionamiento que asume el trabajador frente a lo prescripto, a partir de su expertise y de un contexto de desempeño favorable. Implica, entonces, la posibilidad de advertir anticipadamente desvíos, resolver problemas o "eventos" e introducir acciones correctivas considerando criterios preestablecidos, pero sin necesidad de una supervisión estrecha.

El contexto de desempeño favorece la autonomía cuando: a) el trabajador dispone de cierta expertise para participar en las decisiones que atañen a las rutinas de su sector; b) los estilos de conducción imperantes son más formativos que directivos y c) existe un bajo nivel de disciplinamiento de las interacciones y el ambiente físico y social de trabajo estimula la interconsulta y cooperación. La autonomía puede tomar dos direcciones, o bien forma parte del sistema y debe cumplirse al igual que el resto de las normas o bien emerge como parte de un proceso dinámico y permite la resolución de problemas. Este último sentido, asigna a la autonomía un papel motivador y propicia el desenvolvimiento de los procesos de aprendizaje (Mallet, 1995).

A los fines de este estudio, fue posible establecer los rasgos de autonomía a partir de la observación de la participación de los trabajadores en distintas instancias, y de los contenidos sobre los que opera dicha participación. Se calificó entonces de autonomía virtuosa a la situación en que los trabajadores realizan no sólo tareas de control de calidad, sino también de programación y mantenimiento. En contrapartida, la autonomía adopta un carácter restringido cuando la participación se limita al control de calidad y sólo a una parte de las tareas mencionadas. Finalmente, no hay autonomía cuando las actividades del trabajador se restringen al proceso productivo o sólo a la actividad vinculada al control de calidad.

Otro de los elementos tenidos en cuenta en las formas organizacionales se vincula con las características de la participación y las posibilidades que al respecto tienen los trabajadores. En este sentido, una lógica participativa implica que la empresa ofrezca la posibilidad a los trabajadores de integrar equipos de mejora continua y de aportar sugerencias, mostrándose receptiva respecto de los aportes que éstos realizan.

La Tabla 1 muestra la forma en que los trabajadores operativos encuestados desarrollan sus tareas en el marco de los indicadores seleccionados. Se observa que el $54 \%$ realiza su trabajo de manera individual, o bien en equipos conformados por un número elevado de integrantes. En ese mismo sentido, la polivalencia enriquecedora aparece en un porcentaje muy reducido, alcanzando solamente al $12 \%$ de los trabajadores. En lo que se refiere a la participación, el mayor número de operarios se encuentran en un nivel medio. En tanto el $41 \%$ de los trabajadores tiene autonomía baja en la realización de sus tareas. 
Impacto de la organización del trabajo en los procesos formativos del sector automotriz Delfini, Marcelo; Pujol, Andrea y Roitter, Sonia

Tabla 1 Trabajadores según características de la realización de tareas (en \%)

\begin{tabular}{cccccc}
\hline \multirow{2}{*}{ Indicador } & Categorías & \multicolumn{4}{c}{ Tipo de empresa } \\
\cline { 3 - 6 } & & Terminal & Global & No global & Total \\
\hline \multirow{2}{*}{$\begin{array}{c}\text { Realización } \\
\text { de tareas }\end{array}$} & Individual & $35^{* * *}$ & 64 & 57 & 54 \\
& Limitada & 25 & 26 & 27 & 26 \\
& Virtuosa & $39 *$ & 10 & 16 & 20 \\
& Total & 100 & 100 & 100 & 100 \\
Polivalencia & Especializadora & 46 & 67 & 63 & 60 \\
& Funcional & $50^{* * *}$ & 23 & 20 & 28 \\
& Enriquecedora & 4 & 10 & 16 & 12 \\
& Total & 100 & 100 & 100 & 100 \\
& Baja & $111^{*}$ & 34 & 39 & 31 \\
& Limitada & 35 & 49 & 46 & 44 \\
& Alta & $53^{* * *}$ & 17 & $15 *$ & 25 \\
& Total & 100 & 100 & 100 & 100 \\
& Buaja & $25 *$ & 41 & 51 & 41 \\
& Limitada & 43 & 36 & 22 & 32 \\
& Virtuosa & 32 & 23 & 27 & 27 \\
& Total & 100 & 100 & 100 & 100 \\
\hline
\end{tabular}

Fuente: Elaboración propia basada en los datos de la encuesta realizada a los trabajadores de la trama. Nota: ${ }^{* * *}$ test $Z$ significativo al $1 \%,{ }^{* *}$ test $Z$ significativo al $5 \%$, ${ }^{*}$ test $Z$ significativo al $10 \%$. En cada caso se coloca el signo respectivo.

En relación al lugar ocupado en la trama, los indicadores seleccionados en la Tabla 1 muestran que se destacan las terminales por las formas de organización del trabajo en equipos de carácter virtuoso, dado que el $39 \%$ de los trabajadores realizan las tareas bajo esas condiciones. Estas firmas también se destacan por la existencia de rotación funcional y por brindar mayores posibilidades de participación a sus empleados, a diferencia de las proveedoras, sobre todo de las empresas no globales. También esta Tabla 1 permite observar la fuerte heterogenei- dad existente entre las diferentes firmas y al interior de las mismas, dando cuenta de la fuerte hibridación existente en la adopción de los modelos productivos y las formas organizacionales correspondientes.

\section{La organización del trabajo y sus configuraciones}

La conformación de clusters permitió avanzar en el análisis de la información y profundizar en la comprensión de las características de la organización del 
trabajo y su relación con los procesos formativos. En esta instancia se utilizó una técnica de análisis exploratorio multidimensional: el Análisis Factorial de Correspondencias Múltiples (AFCM), que permite constituir grupos homogéneos (Clusters) considerando simultáneamente las diferentes características que se pretende estudiar. El AFCM permite analizar todas las asociaciones existentes entre las diferentes modalidades de las variables que componen una matriz de datos y obtener luego, a partir de ello, un conjunto de clases compuestas, en este caso, por trabajadores que presentan una alta homogeneidad intragrupo y una elevada heterogeneidad extragrupo (Lebart, 1998 y Roitter et al., 2008).

Para interpretar correctamente los resultados del análisis de cluster, debe tenerse en cuenta que las modalidades de las variables que se encuentran asociadas a un determinado grupo indican que los individuos que tienen esa característica poseen una representación en el grupo que es superior (significativamente) a la que tienen en el total de la muestra, lo cual no necesariamente implica que todas los trabajadores de dicho grupo presenten esa característica.
Para la construcción de los clusters se tuvieron en cuenta los siguientes indicadores: la forma que adquiere la realización de tareas, la polivalencia de los trabajadores, el nivel de autonomía y las características que adquieren las instancias de participación. Quedaron entonces constituidos cuatro grupos homogéneos, luego integrados en dos, considerando los elementos "estructurales" y de "gestión social". En este sentido estos dos grupos pueden clasificarse en simples y complejos y dividirse a su vez, cada uno de ellos, en limitado y virtuoso (Ver Figura 1).

El primero de los grupos, definido como "simple limitado" se caracteriza por la realización individual de tareas, sin rotación de los trabajadores, un nivel de autonomía bajo y escasa participación de los trabajadores.

El segundo de los grupos se caracteriza por un nivel de autonomía y participación medio, por lo cual quedó definido como "simple virtuoso", considerando que la organización incorpora en su estructura pocos aspectos vinculados a las organizaciones formativa, alcanzándose apenas niveles intermedios de desarrollo.

El tercer grupo, denominado "complejo limitado", se caracteriza por un ele-

Figura 1. Conformación de los clusters

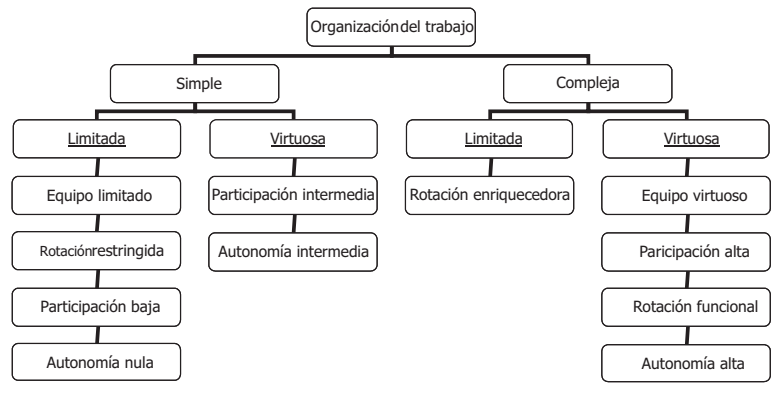

Fuente: Elaboración propia. 
Impacto de la organización del trabajo en los procesos formativos del sector automotriz Delfini, Marcelo; Pujol, Andrea y Roitter, Sonia

vado nivel de rotación -por encima de los otros grupos-. Sin embargo, no se observan otros aspectos virtuosos o característicos de las organizaciones formativas.

El cluster "complejo virtuoso", en cuarto lugar, se estructura en torno a las características propias de los equipos virtuosos: trabajo en equipo con número adecuado de integrantes, participación elevada de los trabajadores, alta autonomía y rotación de carácter funcional, acercándose a aquellas estructuras consideradas en la literatura como de mayor carácter formativo, donde se integran los rasgos estructurales y los aspectos vinculados a una "gestión social" de la organización.

Las consideraciones aquí expuestas y los resultados obtenidos en la exploración, permiten inferir que la comprensión del papel que juega la organización del trabajo en el desarrollo de competencias organizacionales y personales constituye una tarea compleja que exige de progresivos estudios específicos. En el caso que nos ocupa, y en el marco de las posibilidades que nos brindan las herramientas escogidas, es posi- ble realizar una aproximación a la problemática evidenciando sus aspectos más resaltantes y particularizando en las dimensiones de la organización del trabajo que impactan más significativamente sobre los saberes de los trabajadores. Por otro parte, la evidencia empírica que se presenta a partir de la constitución de los grupos homogéneos, permite superar la dicotomía entre formas de organización del trabajo tayloristas y las asociadas a los modelos de "lean production" estableciendo la heterogeneidad de situaciones presentes en los espacios de trabajo, acercándose de esta manera a los estudios recientes desarrollados en Europa (Lorenz y Valeyre, 2005).

El primero de los análisis realizados luego de la constitución de los clusters (Tabla 2), permite observar que el $45 \%$ de los trabajadores de la trama realiza sus tareas bajo una lógica de carácter "simple limitada", mientras que un $20 \%$ lo hace bajo formas de organización "complejas virtuosas". Se destaca que el $43 \%$ de los operarios de las empresas terminales se desempeña dentro de esta lógica, es decir que en el núcleo prevalecen

Tabla 2

Clusters de organización del trabajo por tipo de empresa, en \%

\begin{tabular}{|c|c|c|c|c|c|}
\hline \multirow[b]{2}{*}{$\begin{array}{c}\text { Tipo de } \\
\text { empresa }\end{array}$} & \multicolumn{5}{|c|}{ Cluster } \\
\hline & $\begin{array}{c}\text { Simple } \\
\text { limitada }\end{array}$ & $\begin{array}{c}\text { Simple } \\
\text { virtuosa }\end{array}$ & $\begin{array}{l}\text { Compleja } \\
\text { limitada }\end{array}$ & $\begin{array}{l}\text { Compleja } \\
\text { Virtuosa }\end{array}$ & Total \\
\hline Terminal & 18 & 36 & 3 & $43^{* * *}$ & 100 \\
\hline Global & 51 & 26 & 10 & 13 & 100 \\
\hline No global & 55 & 16 & 17 & $12-^{*}$ & 100 \\
\hline Total & 45 & 24 & 11 & 20 & 100 \\
\hline
\end{tabular}

Fuente: Elaboración propia basada en los datos de la encuesta realizada a los trabajadores de la trama.

Nota: ${ }^{* * *}$ test $Z$ significativo al $1 \%$, ${ }^{* *}$ test $Z$ significativo al $5 \%$, ${ }^{*}$ test $Z$ significativo al $10 \%$. En cada caso se coloca el signo respectivo. 
formas de trabajo en equipos virtuosos, niveles de participación de carácter complejo, una rotación funcional entre puestos y niveles altos de autonomía, situación opuesta a la que se observa entre los proveedores no globales. Es decir, en las firmas terminales los trabajadores estarían realizando sus tareas bajo formas organizacionales complejas que suponen una mayor incorporación de saberes y competencias en instancias laborales.

Una dimensión diferente de la autonomía, referida a las formas de resolución de problemas, puede ser analizada como elemento complementario a la autonomía sustentada en la realización de las tareas. Al respecto, la Tabla 3 evidencia las diferentes dimensiones de la autonomía para resolver los problemas y su desarrollo según los clusters. Las organizaciones más complejas se destacan por la presencia de los rasgos de menor y mayor autonomía simultáneamente, po- niendo en evidencia las formas hibridadas que puede adquirir la organización del trabajo y también que un alto nivel de codificación de las prácticas puede estar evidenciando pérdida de autonomía. Al respecto, si bien el número total de trabajadores que poseen una elevada autonomía en la resolución de problemas es del $(6.6 \%)$, en la forma compleja virtuosa se destaca por llegar al $23 \%$ de los casos. Por otra parte, también en este tipo de organizaciones se desarrollan formas menos autónomas de resolución de problemas. La Tabla 3 pone en evidencia los niveles de este tipo de autonomía que se desarrolla en la empresa y manifiesta la contracara de los discursos empresarios en torno a la posibilidad de los trabajadores de aplicar sus saberes al proceso de trabajo en la medida que se privilegia la mirada constante del supervisor sobre las condiciones de resolución de problemas. Así lo muestran las dos primeras colum-

Tabla 3

Autonomía en la resolución de problemas según cluster, en $\%$

\begin{tabular}{ccccccc}
\hline \multicolumn{7}{c}{$\begin{array}{l}\text { Qué espera la empresa que Ud. haga frente a problemas que aparecen } \\
\text { frecuentemente }\end{array}$} \\
\hline Cluster & $\begin{array}{c}\text { Llamar al } \\
\text { supervisor } \\
\text { para que lo } \\
\text { resuelva }\end{array}$ & $\begin{array}{c}\text { Antes de } \\
\text { emprender la } \\
\text { solución, } \\
\text { consultar al } \\
\text { supervisor }\end{array}$ & $\begin{array}{c}\text { Resolverlo y } \\
\text { luego avisarle } \\
\text { al supervisor }\end{array}$ & $\begin{array}{c}\text { Resolverlo } \\
\text { sin } \\
\text { necesidad } \\
\text { de avisarlo }\end{array}$ & $\begin{array}{c}\text { Buscar la } \\
\text { solución y } \\
\text { prevenir los } \\
\text { problemas }\end{array}$ & Total \\
\hline Simple Limitada & 14 & 47 & 30 & 6 & 3 & 100 \\
Simple Virtuosa & 27 & 53 & 20 & 0 & 0 & 100 \\
Compleja limitada & 0 & 67 & 33 & 0 & 0 & 100 \\
Compleja virtuosa & $46{ }^{*}$ & 15 & 8 & 8 & $23^{* * *}$ & 100 \\
Total & 23 & 42 & 23 & 5 & 7 & 100 \\
\hline
\end{tabular}

Fuente: Elaboración propia basada en los datos de la encuesta realizada a los trabajadores de la trama. Nota: ${ }^{* * *}$ test $Z$ significativo al $1 \%,{ }^{* *}$ test $Z$ significativo al $5 \%,{ }^{*}$ test $Z$ significativo al $10 \%$. En cada caso se coloca el signo respectivo. 
Impacto de la organización del trabajo en los procesos formativos del sector automotriz Delfini, Marcelo; Pujol, Andrea y Roitter, Sonia

nas, las cuales suman el $65 \%$ de los casos, que ante problemas acontecidos en la producción deben o bien llamar al supervisor o consultarlo con él.

Con respecto a la situación contractual en que se encuentran los trabajadores (ver Tabla 4) se observa que la cantidad de personal efectivo se encuentra por debajo del promedio del panel en las dos formas limitadas y por encima en las formas complejas.

En cuanto a los salarios, se puede resaltar que los niveles más altos corresponden a las organizaciones virtuosas, tanto simples como complejas. De hecho, una proporción significativamente mayor de las organizaciones complejas virtuosas paga salarios de más de $\$ 2.200$, mientras que el $48 \%$ de los trabajadores que se desempeñan en organizaciones "simples limitadas" perciben salarios que no superan los \$1.200 (Tabla 5).

En lo que hace al tamaño de las empresas, puede destacarse que en la forma "simple limitada" sobresalen las firmas medianas, que emplean entre $101 \mathrm{y}$ 500 personas, mientras que en la lógica "compleja virtuosa" el número de emplea-

Tabla 4

Situación contractual según cluster, en $\%$

\begin{tabular}{lccc}
\hline \multicolumn{1}{c}{ Cluster } & Efectivo & $\begin{array}{c}\text { Situación contractual } \\
\text { Contrato a plazo }\end{array}$ & Total \\
\hline Simple limitada & 75 & $25^{*}$ & 100 \\
Simple virtuosa & 90 & $10-^{*}$ & 100 \\
Compleja limitada & 69 & $31^{*}$ & 100 \\
Compleja virtuosa & $91^{*}$ & 9 & 100 \\
Total & 81 & 19 & 100 \\
\hline
\end{tabular}

Fuente: Elaboración propia basada en los datos de la encuesta realizada a los trabajadores de la trama.

Nota: ${ }^{* * *}$ test $Z$ significativo al $1 \%,{ }^{* *}$ test $Z$ significativo al $5 \%,{ }^{*}$ test $Z$ significativo al $10 \%$. En cada caso se coloca el signo respectivo.

Tabla 5

Salarios según cluster, en \%

\begin{tabular}{lcccccc}
\hline & \multicolumn{5}{c}{ Salarios } \\
\cline { 2 - 6 } \multicolumn{1}{c}{ Cluster } & Hasta $\mathbf{1 2 0 0}$ & de $\mathbf{1 2 0 0}$ a1800 & de 1800 a 2200 & Más de 2200 & Total \\
\hline Simple limitada & $48^{*}$ & 24 & 22 & 6 & 100 \\
Simple virtuosa & 19 & $50 *$ & 4 & $27^{*}$ & 100 \\
Compleja Limitada & 38 & $54^{*}$ & 0 & 8 & 100 \\
Compleja virtuosa & 23 & 18 & 27 & $32^{* *}$ & 100 \\
Total & 36 & 32 & 16 & 16 & 100 \\
\hline
\end{tabular}

Fuente: Elaboración propia basada en los datos de la encuesta realizada a los trabajadores de la trama. Nota: ${ }^{* *}$ test $Z$ significativo al $1 \%$, ${ }^{* *}$ test $Z$ significativo al $5 \%$, ${ }^{*}$ test $Z$ significativo al $10 \%$. En cada caso se coloca el signo respectivo. 
dos supera los 1500. En este marco, llama la atención el grupo "complejo limitado" compuesto por trabajadores de empresas de menor porte (Tabla 6).

De los análisis precedentes puede inferirse que los trabajadores del panel desarrollan sus tareas en contextos organizacionales heterogéneos. Sin embargo, puede destacarse que los trabajadores de las empresas terminales se integran en procesos más próximos a aquellos que caracterizan a las empresas modernas y que posibilitan un desarrollo relativamente virtuoso de las competencias laborales.

En este sentido, una estrecha vinculación de los cambios de los procesos productivos con el desarrollo de competencias de los trabajadores impactaría en un incremento efectivo en la productividad del trabajo.

No obstante, esta situación no es la más frecuente ya que se observan contradicciones relevantes entre el discurso de la empresa y los comentarios de los trabajadores, que evidencian que el criterio de intensidad y eliminación de tiempos muertos aparece como fenómeno central, inclusive con repercusiones sobre la calidad de los productos y procesos.

"Si yo tengo un problema y estoy haciendo una refacción y veo que no lo puedo terminar, tengo que parar la línea sí o sí... pero ahí tengo que priorizar... si conviene parar la línea, porque sé que no voy a parar más de 10 segundos, o dejar seguir el problema. Hay una planilla que acompaña al vehículo en la cual se declara..: 'falta el bulón del levanta vidrios porque... se cortó..', y mi nombre. Tiene que ir el responsable" (Operadorlíder Terminal).

Estas lógicas restrictivas se profundizan cuando los aumentos de la producción imponen nuevos puestos de trabajo. Así lo corrobora el operador líder en la frase que sigue:

"Se agregan puestos de trabajo. Se hacen menos cosas dentro de tu puesto. Si el operario que arma... la puerta en 2 minutos pone: el levanta vidrios, el vidrio de la puerta delantera, en 2 minutos $y$ pico, si sólo va a poner el levanta vidrios, y el vidrio lo ponen en la operación siguiente,(/). Pero qué pasa... a él la puerta

Tabla 6

Ocupados según cluster, en \%

\begin{tabular}{lccccc}
\hline & \multicolumn{5}{c}{ Ocupados } \\
\cline { 2 - 7 } \multicolumn{1}{c}{ Cluster } & Menos de $\mathbf{1 0 0}$ & Entre $\mathbf{1 0 1}$ y $\mathbf{5 0 0}$ & Entre $\mathbf{5 0 1}$ y $\mathbf{1 5 0 0}$ & Más de $\mathbf{1 5 0 0}$ & Total \\
\hline Simple limitada & 13 & $54^{*}$ & 29 & $4-^{* * *}$ & 100 \\
Simple virtuosa & $3-^{*}$ & 32 & $44^{*}$ & 21 & 100 \\
Compleja limitada & $46^{* * *}$ & 46 & 0 & 8 & 100 \\
Compleja virtuosa & 13 & 22 & 22 & $43^{* * *}$ & 100 \\
Total & 15 & 41 & 28 & 16 & 100 \\
\hline
\end{tabular}

Fuente: Elaboración propia basada en los datos de la encuesta realizada a los trabajadores de la trama.

Nota: ${ }^{* * *}$ test $Z$ significativo al $1 \%,{ }^{* *}$ test $Z$ significativo al $5 \%,{ }^{*}$ test $Z$ significativo al $10 \%$. En cada caso se coloca el signo respectivo. 
Impacto de la organización del trabajo en los procesos formativos del sector automotriz Delfini, Marcelo; Pujol, Andrea y Roitter, Sonia

no le va a pasar... cada 2 ... ahora le va a pasar cada un minuto y medio. En vez de armar..., ahora son 50 vehículos por día,... son 100 puertas que él arma. El año que viene van a ser alrededor de 100 vehículos por... turno...entonces le van a pasar 200 puertas" (operador líder).

Precisamente, la división excesiva de las tareas produce efectos especializadores sobre el trabajador, en la medida en que limita la rotación al profundizarse la división técnica.

Además, las condiciones organizacionales en que los trabajadores desarrollan sus tareas indicarían la preferencia, por parte de las firmas, de trabajadores con un alto grado de especialización y con niveles de autonomía restringidos a ciertos espacios, alejándose de esta manera de los discursos que expresan la necesidad del desarrollo de trabajadores autónomos, de carácter polivalente y participativos en las empresas.

Si bien no se desarrolla en extenso en este artículo, cabe destacar que el proceso de construcción de clusters permitió además el análisis de algunos aspectos de la organización del trabajo que resultan de importancia para la comprensión global de la problemática (Pujol, Roitter y Delfini, 2006; 2007). En el caso de la capacitación por ejemplo, se observó que el $66 \%$ de los trabajadores no han recibido capacitación o recibieron menos de 8 horas al año. Los niveles altos de capacitación ${ }^{4}$ sólo fueron alcanzados por el $5,6 \%$ de los operarios.
En cuanto a los contenidos de la capacitación recibida por el $78 \%$ de los trabajadores, los temas se limitaron sólo a normas de seguridad y de calidad.

También pudo observarse menor presencia de procesos de evaluación de desempeño en las formas organizacionales de carácter "simple limitado" en las que no se desarrolla una evaluación periódica de los trabajadores.

Por otra parte, el carácter participativo de los trabajadores en la empresa y las formas de comunicación se revelan en las características que presentan los sistemas de sugerencias. Se evidenció que una alta proporción de los trabajadores $(25,7 \%)$ no ha participado en los sistemas de sugerencias o bien no existen en la empresa o sector donde trabajan.

\section{Conclusiones}

Los resultados obtenidos en este estudio permiten realizar un conjunto de consideraciones relevantes, algunas de ellas de carácter más general, referidas al "trabajo" y otras más específicas en torno a la problemática de los "trabajadores".

Desde una perspectiva global, las diferencias existentes entre los resultados obtenidos en el núcleo de la trama y los de empresas proveedoras dan lugar al análisis de los procesos de transferencia de tecnología y circulación de conocimiento de la trama misma, por un lado, y al análisis del modo en que su optimiza-

4 El indicador de capacitación se construyó de la siguiente manera: Capacitación 1, es aquella donde el trabajador no recibió o recibió menos de 8 horas de capacitación. Capacitación 2, es la recibida entre 8 y 34 horas. La capacitación 3 es la recibida por más de 34 horas. 
ción podría incidir en la competitividad dinámica y en el desarrollo de las competencias organizacionales y de los trabajadores, por el otro.

En la situación descripta, es posible suponer que las empresas núcleo de la trama se ven favorecidas por la interacción en redes corporativas y la transferencia de tecnología de gestión desde sus casas matrices. No obstante, estos procesos parecen no trasladarse a los proveedores, lo que desde una perspectiva sistémica necesariamente implica cierta pérdida de efectividad para el conjunto.

Este fenómeno muestra, en parte, los alcances de la implementación de los sistemas flexibles a nivel local, que parecen limitarse a la incorporación de algunas o muchas de sus herramientas pero sin que se evidencie una innovación profunda o cambio paradigmático en relación con los "modos de hacer". En este sentido, por ejemplo, al igual que la implementación de nuevas tecnologías y nuevas formas de organización del trabajo, el desarrollo de proveedores por parte de las terminales ${ }^{5}$ constituye un componente clave de la optimización de la trama y constituye un indicador sensible respecto del nivel de penetración de los sistemas de producción flexible en la realidad local. Estos elementos, pueden explicarse por los rasgos de la trama automotriz argentina específicamente porque en ella el desenvolvimiento de los vínculos entre firmas se basa en las formas de imposición de las firmas núcleo sobre sus proveedores más que sobre fenómenos de cooperación entre las diferentes firmas componentes de la trama, mecanismo este último, que daría lugar a la formación de estructuras organizativas de mayor virtuosismo, ya que los procesos innovativos podrían sustentarse en la cooperación con la intervención de los diferentes actores del trabajo.

Otra explicación posible implica la consideración de que no se trata de un proceso de modernización parcial, sino de modernización incompleta o inconclusa. De acuerdo con esta línea de análisis, se trataría de un cambio que no alcanzó a consolidarse y que tras un proceso de recesión y crisis, actualmente encuentra dificultades para completarse porque el ritmo de la reactivación productiva no sólo lo impide sino que además, y por el contrario, moviliza hacia una re-taylorización.

La evidencia de la existencia de organizaciones más simples en las firmas proveedoras respecto de las firmas terminales no sólo impacta a nivel de las competencias organizacionales. Esta diferencia permite interpretar que el trabajo en las firmas del núcleo de la trama, al ser más rico, ofrecería un contexto más adecuado para el desarrollo de los trabajadores, con lo que esta diferencia "técnica" de organización deriva en una diferencia "social", en tanto la movilidad ocupacional hacia el grupo virtuoso no sólo implica una mejora salarial, sino también la ad-

$5 \quad$ Este aspecto es más significativo aún en la versión japonesa de los sistemas flexibles, la que se caracteriza por una política más activa respecto del desarrollo de proveedores que la que se observa en otras aplicaciones (Francia, Italia, Alemania). 
Impacto de la organización del trabajo en los procesos formativos del sector automotriz Delfini, Marcelo; Pujol, Andrea y Roitter, Sonia

quisición de mayor profesionalidad; en contrapartida, los trabajadores que permanecen en estas firmas encuentran un techo a la hora de desarrollar nuevas capacidades. En este sentido, cuando las empresas núcleo de la trama no traccionan el cambio en los modos de hacer de las firmas proveedoras, la dinámica tiende a la reproducción de las diferencias.

En un análisis más específico respecto de la relación entre organización del trabajo y desarrollo de competencias laborales, los datos muestran también una distancia considerable entre el diseño de la organización del trabajo y su puesta en acción. Los resultados que arrojan los indicadores referidos a participación, polivalencia, rotación y autonomía -y claramente los indicadores básicos de capacitación- no revelan el predominio de una lógica formativa en las políticas de gestión de mano de obra, impresión que se refuerza al integrar al análisis la voz de los trabajadores.

Por otra parte, al introducir la variable del tamaño de la empresa, se pudo establecer la existencia de organizaciones "complejas virtuosas" en las firmas de mayor tamaño, lo que ayudaría al desarrollo de procesos de interacción y al desenvolvimiento de lógicas de aprendizaje basadas en la imitación y la experiencia. Sin embargo, ello se ve restringido por la limitación que se produce en las formas de autonomía dinámica, donde surgen diferentes lógicas de su desarrollo. Esto permite inferir la existencia de limitantes a los procesos de aprendizaje en la empresa.

Siguiendo el primer análisis general antes expuesto, esto puede deberse a un proceso de modernización parcial y fragmentario, más orientado a prescribir nuevos comportamientos desde una renovación del lenguaje -como una forma más elaborada de disciplinamiento- que a construir una comunidad de prácticas genuinas en la que sea posible el aprendizaje: un cambio técnico sin cambio en los supuestos sociales de base.

En el marco de esta lectura, las expresiones de los trabajadores enfatizan la importancia del "tiempo cronológico" y de la intensidad del trabajo por sobre el tiempo lógico del aprendizaje, junto con otro tipo de prácticas características de sistemas simples; por lo que cabe interrogarse si se trata de una característica coyuntural o estructural del desenvolvimiento de las firmas. En cualquiera de los casos, asistimos a un escenario escasamente propicio para el incremento de empleo de calidad y la mejora de la competitividad.

\section{Referencias Bibliográficas}

Arundel, Anthony; Lundvall, Bengt Ake.; Lorenz, Edward and Valeyre, Antoine (2007). How Europe's economies learn: a comparison of work organization and innovation mode for the EU15, Industrial and Corporate Change, Oxford University Press, vol. 16(6), pages 1175-1210, December.

Arundel, Anthony; Lundvall, Bengt Ake; Lorenz, Edward and Valeyre, Antoine (2003). "Organisational of work and innovative performance: A comparison of the EU-15". DRUID, Working Paper $\mathrm{N}^{\circ} 6-14$.

Antunes, Ricardo (2006). Riqueza e miseria do trabalho no Brasil. Sao Paulo. Boitempo Editorial.

Argyris, Chris y Schon, Donald (1978). Organizational Learning: A theory of action perspective. USA, AdissonWesley, Readin, Mass. 
Arocena, Rodrigo (1996). Los pequeños países periféricos ante la revolución tecnológica: un marco preliminar para la discusión del tema. Revista de Ciencias Sociales, $N^{\circ} 12$. Montevideo. Universidad de la República. Facultad de Ciencias Sociales.

De La Garza, Enrique (1999). Los retos teóricos de los estudios del trabajo hacia el siglo XXI. Buenos Aires. Grupos de Trabajo CLACSO.

Erbes, Analía; Robert, Verónica; Yoguel, Gabriel; Borello, José y Llebedinsky, Viviana (2006). Regímenes tecnológicos, de conocimiento y competencia en diferentes formas organizacionales: la dinámica entre difusión y apropiación. En: Desarrollo Económico. Revista de ciencias sociales, Vol. $46, N^{\circ} 181$. Buenos Aires, IDES.

Gore, Ernesto (2003). Conocimiento colectivo. La formación en el trabajo y la generación de capacidades colectiva. Buenos Aires, Garnica.

Harteis, Chistian (2003). La organización autoformativa desde la perspectiva de sus empleados. En: Revista Europea de formación profesional, $\mathrm{N}^{\circ} 29$. París, Cedefop, pp. 18-28.

Hautchuel, Armand, y Weil, Benoit (1999). "Design-Oriented Organisations, Towards a Unified Theory of Design Activities". 6th international product development management conference, Reino Unido, Churchill College, Cambridge.

Linhart, Daniele (2002). "Los asalariados y la mundialización”, Sociología del Trabajo, nueva época $\mathrm{N}^{\circ} 45$.

Lorenz, Edward y Valeyre, Antoine (2005). Organisational Innovation, Human Resource Management and Labour Market Structure: A Comparison of the EU-15, En: Journal of Industrial Relations, Volume 47, Issue 4, Decem- ber 2005 Sidney, SAGE Publications, pp 424-442.

Lundvall, Bengt Ake (2003). ¿Por qué la nueva economía es una economía del conocimiento? En Boscherini, Novick y Yoguel (Eds) Nuevas tecnologías de información y comunicación: los límites en la economía del conocimiento, Madrid-Buenos Aires, Editorial Miño y Dávila.

Lundvall, Bengt-Ake (2009). The Danish Model and the Globalizing Learning Economy: Lessons for Developing Countries, Working Papers UNU-WIDER Research Paper, World Institute for Development Economic Research (UNU-WIDER).

Lebart, Ludovic; Morineau, Alain y Piron, Marie (1998). Statique Exploratoire Multidimensionnelle. Dunnot- París.

Lundvall, Bengt Ake (1988). Innovation as an interactive process -from user- producer interaction to the national system of innovation. En: Dosi, G, (eds,), Technical change and economy theory, Londres, Pinter Publishers.

Mallet, Louis (1995). Organización formativa, coordinación y motivación. En: Revista Europea de formación Profesional, No 5. París. Cedefop, pp 11-17.

Méhaut Philippe (1994). Transformaciones organizativas y políticas de formación: ¿Cuáles son las lógicas de las competencias? En: Revista Europea de formación Profesional, № 1. París. Cedefop, pp 56-64.

Mello e Silva, Leonardo (2004). Trabalho em grupo e sociabilidade privada. Sao Paulo. Editora 34.

Mertens, Leonard (2002). Formación, productividad y competencia laboral en las organizaciones, Montevideo, OIT. Centro Interamericano de Investigación y Documentación sobre Formación Profesional. 
Impacto de la organización del trabajo en los procesos formativos del sector automotriz Delfini, Marcelo; Pujol, Andrea y Roitter, Sonia

Monterio Leite, Elenice (1996). El rescate de la calificación, Montevideo CINTERFOR.

Motta Jorge; Roitter, Sonia; Delfini, Marcelo; Yoguel, Gabriel y Milesi, Darío (2007). Articulación y desarrollo de competencias en la trama automotriz argentina: morfología, innovación y empleo. En: Delfini, M.; Dubbini, D.; Lugones, M. y Rivero, I. (Coords.) Innovación y empleo en tramas productivas de Argentina, Buenos Aires, Prometeo, pp.231-274.

Nelson, Richard y Winter, Sidney (1982). An evolucionary of economic change, Cambridge, Harvard University Press, M.A.

Nonaka, Ikuhiro y Takeuchi, Hirotaka (1999). La organización creadora de conocimiento. Cómo las compañías japonesas crean la dinámica de la innovación, México, Oxford.

Novick, Marta (2000). La transformación en la organización del trabajo. En: De La Garza Toledo, E (Coord.) Tratado Latinoamericano de sociología del trabajo, México FCE, pp. 123-147.

Parlier, Michel (1996). De l'entreprise qui forme à l'entreprise qui apprend. Rev. Actualité de la formation permanente. $N^{\circ} 143$. Dossier: París, Du référentiel emploi au référentiel formation, pp 10-18.

Pujol, Andrea (2000). Percepciones acerca de la innovación tecnológica en los trabajadores del sector lácteo. Una mirada desde el saber obrero. Jornadas de la Facultad de Psicología. Universidad Nacional de Córdoba.

Pujol, Andrea (2001). La reconversión productiva y la capacitación del personal en el sector lácteo. En: Revista Venezolana de Gerencia Año 6 NN$^{\circ} 13$. Maracaibo. Venezuela, Universidad de Zulia.

Pujol, Andrea; Roitter, Sonia y Delfini, Marcelo (2006) ¿La organización del trabajo como espacio formativo? El caso de la trama productiva automotriz argentina. En: V Congreso Latinoamerica- no de Sociología del Trabajo. Abril de 2007, Montevideo.

Roitter, Sonia; Erbes, Analía; Gabriel Yoguel, Delfini, Marcelo y Pujol, Andrea (2008) "Competencias endógenas y vinculaciones en agentes pertenecientes a las tramas productivas automotriz y siderúrgica" En: Revista Economía: Teoría y Práctica, Universidad Autónoma Metropolitana, Ciudad de México. pp. 69-118

Rullani, Enzo (2006). "El valor del conocimiento", in Di Maria E., Micelli S. (a cura di), Sistemas locales en las redes internacionales de produccion, Aracne, Roma, 2006, pp. 15-46.

Rullani, Enzo (2000). El valor del conocimiento. En Boscherini y Poma (coord..), Territorio, conocimiento competitividad de las empresas. El rol de las instituciones en el espacio global, Buenos Aires, Miño y Dávila, pp 229-258.

Yoguel, Gabriel; Novick, Marta; Catalano, Ana y Alabornoz, Facundo (2002). Adaptación de modelos productivos en países emergentes: el caso de la industria automotriz en la Argentina. Universidad Nacional de General Sarmiento. Laboratorio de Investigaciones sobre Tecnología, Trabajo, Empresas y Competitividad; Documentos de Trabajo, Buenos Aires.

Yoguel, Gabriel y Novick, Marta (2000). La vulnerabilidad de una trama productiva: la dificil relación cliente-proveedor en el complejo automotriz argentina. En: Revista Trabajo, Número 4, Invierno. México.

Zarifian, Philippe (1995). La organización autoformativa y el modelo de competencias: ¿qué motivos? ¿qué aprendizajes? En: Revista Europea de formación Profesional, № 5. París. Cedefop, pp 5-10.

Zarifian, Philippe (1996). Travail et comunication. Essai sociologique sur le travail dans la grande entreprise industrielle, París, PUF. 